\title{
Mediators of vascular leak in dengue infections
}

\author{
Malavige $\mathbf{G} \mathbf{N}^{1}$ \\ Journal of the Ceylon College of Physicians, 2015, 46, 7-10
}

\begin{abstract}
Although plasma leakage is the hallmark of severe dengue infections the factors that cause increased vascular permeability have not been identified. As platelet activating factor (PAF), lipid mediators and cytokines are associated with increase in vascular permeability, we set out to investigate the role of these mediators in acute dengue infection.

Levels of inflammatory lipid mediators and cytokines were initially evaluated in 25 patients with confirmed acute dengue infection and 12 healthy individuals. After determining which inflammatory mediators were associated with severe dengue, serial assays of the above lipid mediators, and cytokines were done in an additional 36 patients in serum samples collected 12 hours apart throughout the course of their hospital stay.
\end{abstract}

We found that PAF levels were significantly higher in patients $(p=0.001)$ when compared to healthy individuals and that PAF levels rose just before the onset of the critical phase. PAF values of $>100 \mathrm{ng} / \mathrm{ml}$ were associated with reduced expression of gap junction proteins leading to increased vascular permeability. A similar effect was seen with serum from patients with dengue infection which was reversed by a PAF receptor antagonist. S1P levels which are associated with increasing the integrity of the endothelial barrier were significantly reduced in patients with DHF. Serum IL-10, TNF $\alpha$ and IL-1 $\beta$ levels were elevated in patients with DHF and followed the same patterns as PAF. In conclusion, our results show that PAF is likely to be a potent mediator of vascular leak in dengue and use of PAF blockers could be therapeutically useful.

\section{Introduction}

Dengue viral infection has become one of the most important mosquito borne viral infections in the world

\footnotetext{
${ }_{1}^{1}$ Director, Centre for Dengue Research, Department of Microbiology, Faculty of Medical Sciences, University of Sri Jayewardenepura, Sri Lanka. E-mail: neelikamalavige@gmail.com
}

and is one of the major emerging infectious diseases. It has been predicted that 390 million dengue infections occur per year resulting in approximately 96 million clinically apparent infections. ${ }^{1}$ As a result of the high disease burden due to dengue infections, it has been declared a priority infection by the WHO, UNICEF and World Bank. ${ }^{2}$

Sri Lanka has had several dengue epidemics for the past 21 years, and the incidence and severity of these epidemics is increasing. ${ }^{3}$ The incidence of dengue has been particularly high since 2009 (Figure 1) and the scarce resources available in most hospitals in Sri Lanka have been stretched to the limits. Early detection of shock and other complications and supportive therapy has shown to reduce the morbidity and mortality. ${ }^{4,6}$ The case fatality rates which were $0.99 \%$ in year 2009 and $0.74 \%$ in year $2010^{7}$ has been halved in more recent years due to the enormous efforts taken by physicians, paediatricians and other health care personnel.

Currently there are no effective antiviral drugs to treat acute infection, nor an effective vaccine to prevent infection. However, with early detection of complications and proper fluid management, case fatality rates can be kept very low. Although Sri Lanka and many other countries have achieved this with tremendous effort, it is a significant burden on resource poor hospitals such

\section{Incidence of dengue infections in Sri Lanka}

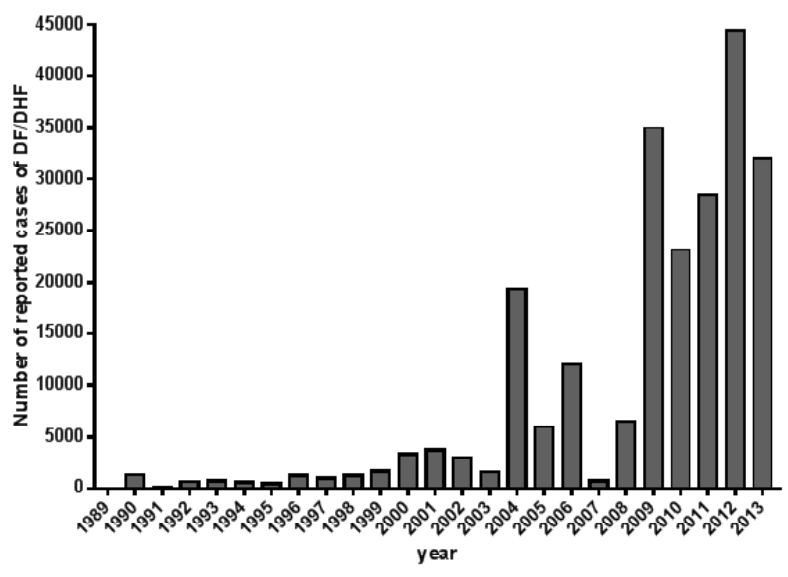

Figure 1. Incidence of dengue infections in Sri Lanka from 1989 to 2013. Source: Sri Lanka Epidemiology unit 
as ours. Therefore, early identification of patients who are likely to develop severe disease and complications would enable clinicians to act quickly in order to minimize severe disease.

\section{Vascular leak in dengue infection}

The dengue virus (DV) potentially infects many cell types ${ }^{8}$, and disseminates around the body in the viraemic phase (febrile phase) which may last for 2-7 more days ${ }^{2}$. The patient may then progress to the critical phase which is characterized by plasma leakage. ${ }^{4}$ Complications as a result of plasma leakage such as shock, pleural effusions, ascites along with other complications such as liver failure and encephalopathy may occur during the critical phase. ${ }^{2}$ The critical phase usually lasts for $24-48$ hours before the patient enters the recovery phase. Some patients however, enter the recovery phase without progressing through the critical phase.

Many factors are thought to play a role in causing endothelial dysfunction in dengue infections. Cytokines and other mediators produced by monocytes have shown to act on the endothelium and cause increased vascular permeability. ${ }^{9,11}$ In addition, in vitro studies have shown that mediators released from mast cells also cause endothelial activation and increased vascular permeability, especially in the presence of dengue immune sera ${ }^{12,13}$ Although many mediators have been implicated as possible causes of endothelial dysfunction and vascular leakage, these studies have been based on in vitro data.

\section{Mediators that cause vascular leak}

Many mediators such as platelet activating factor (PAF), tumour necrosis factor alpha (TNF $\alpha$ ), vascular endothelial growth factor (VEGF), sphingosine 1 phosphate (S1P), monocyte chemo attractant protein -1 (MCP-1) and thrombin have been shown to cause increased vascular permeability in sepsis and anaphylactic shock. ${ }^{14,17}$ The factors that cause vascular leak are thought to be present in serum of dengue patients, as sera from dengue patients have shown to reduce expression of gap junction proteins thus leading to vascular leak. ${ }^{18}$ Among the potential mediators that cause vascular leak, VEGF has been extensively studied in dengue, ${ }^{19,20}$ and it has been shown that free plasma VEGF levels are significantly higher and VEGF receptor 2 levels are significantly lower in those with DHF. $^{20}$ In addition, the rise in VEGF and fall in VEGFR2 was shown to correlate with the onset of vascular leak. ${ }^{20}$ Apart from VEGF, many studies have also shown that TNF $\alpha$ and MCP-1 were increased in DHF, ${ }^{21,22}$ although some studies including studies done by us have shown contradictory results. ${ }^{23,24}$

\section{Platelet activating factor (PAF) as a mediator of vascular leak}

In our most recent studies we evaluated the role of PAF and other lipid mediators in the pathogenesis of vascular leak. PAF is a phospholipid mediator with many biological functions including increasing vascular permeability. ${ }^{25}$ It is rapidly synthesized from many cells such as endothelial cells, leucocytes and monocytes in response to cellular stress. ${ }^{25,26}$ Intravenous administration of PAF in animal experiments was shown to result in hypotension, thrombocytopenia and increased vascular permeability. ${ }^{27,29}$ PAF has also been shown to be associated with vascular leak in mice models of dengue infection, which was reversed by PAF inhibitors called UK-74,505. ${ }^{30}$ In addition, PAFR knockout mice were shown to be less susceptible to developing severe dengue. ${ }^{30}$ Since, PAF was shown to be associated with vascular leak in mice models, we proceeded to determine its role in human dengue infections. We found that PAF levels were significantly elevated in patients with DHF when compared to those with DF, throughout the course of the illness. Since we found that PAF breakdown enzyme levels were also similarly higher in patients with DHF when compared to $D F$, the rise of PAF is likely to be due to increased production. ${ }^{31}$

As PAF levels were higher in patients with DHF and tend to rise just before the onset of the critical phase associated with fluid leakage, we also investigated if PAF indeed caused increased vascular permeability. These experiments were carried out in Human Umbilical Vein Endothelial cell lines (HIVECs) using a PAF agonist, PAF antagonist and serum from patients with dengue infection. The effect of these mediators on HUVECs was determined by assessing the expression of a tight junction protein, ZO-1 by confocal microscopy. ${ }^{32}$ We found that sera from patients with dengue infection resulted in a significant reduction in ZO-1 expression, which was upregulated when the HUVECs were pre-treated with a PAF antagonist. Therefore, these experiments conclusively showed that PAF indeed was a cause of vascular leak in acute dengue. ${ }^{31}$

\section{Sphingosine 1 - phosphate (S1P) as a mediator of vascular leak}

Sphingosines are a family of lipids and when phosphorylated by sphingosine kinases 1 and 2 generate S1P. ${ }^{33,34} \mathrm{~S} 1 \mathrm{P}$ has been shown to protect endothelial barrier integrity induced by VEGF. ${ }^{34}$ S1P antagonists have shown to disrupt endothelial barrier integrity in a dose dependent manner. ${ }^{34}$ In addition, S1P is shown to oppose the inflammatory effects of PAF and reduce vascular leakage induced by PAF in rat models. ${ }^{35}$ In summary, S1P appears to have a 
protective role against vascular leak and maintains endothelial barrier integrity. Therefore, we proceeded to investigate the role of S1P in acute dengue infection. We found that S1P levels were significantly lower in patients with severe forms of dengue. S1P was lowest in those who developed grade IV DHF. ${ }^{36}$ S1P levels particularly reduced during the critical phase in patients with DHF suggesting that a reduction in S1P during the critical phase, is also likely to contribute to increased vascular permeability. ${ }^{36}$

\section{Summary}

Our recent research of the mediators of vascular leak shows that PAF levels were significantly associated with vascular leak. In contrast, S1P which is important in endothelial cell integrity, was detected at lower levels in patients with DHF when compared to $D F$, especially during the critical phase. Drugs that block PAF receptors and those that potentiate the action of S1P are already available and are used for other disease conditions. Therefore, the use of these drugs in the treatment of dengue and their effect on reducing vascular leak should be evaluated.

\section{Acknowledgements}

We are grateful to the Centre for Dengue Research, University of Sri Jayawardenapura and Medical Research Council, UK for funding. I wish to thank Dr. Chandima Jeewandara for the experiments on endothelial cell lines and Mr. Laksiri Gomes for carrying out the cytokine ELISAs. I wish to thank Prof. Graham Ogg from University of Oxford for arranging for confocal microscropy and other inputs. The contribution by my clinical colleagues Dr. Ajantha Liyanage and Dr. Manouri Gamage and the staff of their wards was invaluable. I wish to thank Dr. Shiran Paranavithana, Dr. Nilanka Wickramasinghe, Dr. Samitha Fernando, Dr. Randika H. Fernando and Dr. M. K. Tharindu Perera who tirelessly collected samples 12 hours apart from patients with dengue.

\section{References}

1. Bhatt S, Gething PW, Brady OJ, Messina JP, Farlow AW, Moyes $\mathrm{CL}$, et al. The global distribution and burden of dengue. Nature. 2013; 496(7446): 504-7. Epub 2013/04/09.

2. WHO, editor. Comprehensive guidelines for prevention and control of dengue fever and dengue haemorrhagic fever. SEARO, New Delhi, India: World Health Organization; 2011.

3. Dengue in Sri Lanka. Sri Lanka Epidemiology Unit; 2010 [updated 14/09/2010]; Available from: http://www.epid.gov.lk/ Dengue_updates.htm.

4. WHO. Dengue guidelines for diagnosis, prevention and control. New edition ed. Southeast Asian Office of the WHO World Health Organisation; 2009.

5. Santiago A, Fernandez-Sein A. Dengue in children: critical points in management. Puerto Rico Health Sciences Journal 2001; 20(4): 343-6.

6. Singhi S, Kissoon N, Bansal A. Dengue and dengue hemorrhagic fever: management issues in an intensive care unit. Jornal de Pediatria 2007; 83(2 Suppl): S22-35.

7. Dengue update [database on the Internet]. 2013. Available from: http://www.epid.gov.lk/.

8. Martina BE, Koraka P, Osterhaus AD. Dengue virus pathogenesis: an integrated view. Clinical Microbiology Reviews 2009; 22(4): 564-81.

9. Anderson R, Wang S, Osiowy C, Issekutz AC. Activation of endothelial cells via antibody-enhanced dengue virus infection of peripheral blood monocytes. Journal of Virology 1997; 71(6): 4226-32.

10. Butthep P, Chunhakan S, Yoksan S, Tangnararatchakit K, Chuansumrit A. Alteration of cytokines and chemokines during febrile episodes associated with endothelial cell damage and plasma leakage in dengue hemorrhagic fever. The Pediatric Infectious Disease Journal 2012; 31(12): e232-8. Epub 2012/08/29.

11. Ong SP, Lee LM, Leong YF, Ng ML, Chu JJ. Dengue virus infection mediates HMGB1 release from monocytes involving PCAF acetylase complex and induces vascular leakage in endothelial cells. PloS one 2012; 7(7): e41932. Epub 2012/ 08/04.

12. Brown MG, Huang YY, Marshall JS, King CA, Hoskin DW, Anderson R. Dramatic caspase-dependent apoptosis in antibody-enhanced dengue virus infection of human mast cells. Journal of Leukocyte Biology 2009; 85(1): 71-80.

13. Brown MG, Hermann LL, Issekutz AC, Marshall JS, Rowter $\mathrm{D}, \mathrm{Al}$-Afif $\mathrm{A}$, et al. Dengue virus infection of mast cells triggers endothelial cell activation. Journal of Virology 2011; 85(2): 1145-50.

14. Vadas P, Perelman B, Liss G. Platelet-activating factor, histamine, and tryptase levels in human anaphylaxis. The Journal of Allergy and Clinical Immunology 2013; 131(1): 144-9. Epub 2012/10/09.

15. Bautista AP, Spitzer JJ. Platelet activating factor stimulates and primes the liver, Kupffer cells and neutrophils to release superoxide anion. Free Radical Research Communications 1992; 17(3): 195-209. Epub 1992/01/01.

16. Fisher CJ, Jr., Agosti JM, Opal SM, Lowry SF, Balk RA, Sadoff $\mathrm{JC}$, et al. Treatment of septic shock with the tumor necrosis factor receptor:Fc fusion protein. The Soluble TNF Receptor Sepsis Study Group. The New England Journal of Medicine 1996; 334(26): 1697-702. Epub 1996/06/27.

17. Roviezzo F, Brancaleone V, De Gruttola L, Vellecco V, Bucci M, D'Agostino B, et al. Sphingosine-1-phosphate modulates vascular permeability and cell recruitment in acute inflammation in vivo. The Journal of Pharmacology and Experimental Therapeutics 2011; 337(3): 830-7. Epub 2011/ $03 / 23$.

18. Appanna R, Wang SM, Ponnampalavanar SA, Lum LC, Sekaran SD. Cytokine factors present in dengue patient sera induces alterations of junctional proteins in human endothelial cells. The AmericanJournal of Tropical Medicine and Hygiene 2012; 87(5): 936-42. Epub 2012/09/19. 
19. Rathakrishnan A, Wang SM, Hu Y, Khan AM, Ponnampalavanar S, Lum LC, et al. Cytokine expression profile of dengue patients at different phases of illness. PloS One 2012; 7(12): e52215. Epub 2013/01/04.

20. Srikiatkhachorn A, Ajariyakhajorn C, Endy TP, Kalayanarooj S, Libraty DH, Green S, et al. Virus-induced decline in soluble vascular endothelial growth receptor 2 is associated with plasma leakage in dengue hemorrhagic fever. Journal of Virology 2007; 81(4): 1592-600. Epub 2006/12/08.

21. Bozza FA, Cruz OG, Zagne SM, Azeredo EL, Nogueira RM, Assis EF, et al. Multiplex cytokine profile from dengue patients: MIP-1beta and IFN-gamma as predictive factors for severity. BMC Infectious Diseases 2008; 8: 86.

22. LeeYR, Liu MT, Lei HY, Liu CC, Wu JM, Tung YC, et al. MCP1 , a highly expressed chemokine in dengue haemorrhagic fever/dengue shock syndrome patients, may cause permeability change, possibly through reduced tight junctions of vascular endothelium cells. The Journal of General Virology 2006; 87(Pt 12): 3623-30.

23. Malavige GN, Huang LC, Salimi M, Gomes L, Jayaratne SD, Ogg GS. Cellular and cytokine correlates of severe dengue infection. PloS One 2012; 7(11): e50387. Epub 2012/12/05.

24. Chen LC, Lei HY, Liu CC, Shiesh SC, Chen SH, Liu HS, et al. Correlation of serum levels of macrophage migration inhibitory factor with disease severity and clinical outcome in dengue patients. The American Journal of Tropical Medicine and Hygiene 2006; 74(1): 142-7.

25. Walterscheid JP, Ullrich SE, Nghiem DX. Platelet-activating factor, a molecular sensor for cellular damage, activates systemic immune suppression. The Journal of Experimental Medicine 2002; 195(2): 171-9. Epub 2002/01/24.

26. Valone FH, Epstein LB. Biphasic platelet-activating factor synthesis by human monocytes stimulated with IL-1-beta, tumor necrosis factor, or IFN-gamma. J Immunol 1988; 141(11): 3945-50. Epub 1988/12/01.

27. Carter MB, Wilson MA, Wead WB, Garrison RN. Plateletactivating factor mediates pulmonary macromolecular leak following intestinal ischemia-reperfusion. The Journal of Surgical Research 1996; 60(2): 403-8. Epub 1996/02/01.

28. Walther A, Yilmaz N, Schmidt W, BachA, Gebhard MM, Martin $\mathrm{E}$, et al. Role of platelet-activating factor in leukocyte- independent plasma extravasation and mast cell activation during endotoxemia. The Journal of Surgical Research 2000; 93(2): 265-71. Epub 2000/10/12.

29. Zimmerman GA, McIntyre TM, Prescott SM, Stafforini DM. The platelet-activating factor signaling system and its regulators in syndromes of inflammation and thrombosis. Critical Care Medicine 2002; 30(5 Suppl): S294-301. Epub 2002/05/11.

30. Souza DG, Fagundes CT, Sousa LP, Amaral FA, Souza RS, Souza AL, et al. Essential role of platelet-activating factor receptor in the pathogenesis of Dengue virus infection. Proceedings of the National Academy of Sciences of the United States of America 2009; 106(33): 14138-43. Epub 2009/08/12.

31. Jeewandara C, Gomes L, Wickramasinghe N, GutowskaOwsiak D, Waithe D, Paranavitane SA, et al. Platelet activating factor contributes to vascular leak in acute dengue infection. PLoS Neglected Tropical Diseases 2015; 9(2): e0003459. Epub 2015/02/04.

32. Kanlaya R, Pattanakitsakul SN, Sinchaikul S, Chen ST, Thongboonkerd V. Alterations in actin cytoskeletal assembly and junctional protein complexes in human endothelial cells induced by dengue virus infection and mimicry of leukocyte transendothelial migration. Journal of Proteome Research 2009; 8(5): 2551-62.

33. Rosen H, Goetzl EJ. Sphingosine 1-phosphate and its receptors: an autocrine and paracrine network. Nature Reviews Immunology 2005; 5(7): 560-70. Epub 2005/07/07.

34. Rosen H, Sanna MG, Cahalan SM, Gonzalez-Cabrera PJ. Tipping the gatekeeper: S1P regulation of endothelial barrier function. Trends in Immunology 2007; 28(3):102-7. Epub 2007/02/06.

35. Adamson RH, Sarai RK, Altangerel A, Thirkill TL, Clark JF, Curry FR. Sphingosine-1-phosphate modulation of basal permeability and acute inflammatory responses in rat venular microvessels. Cardiovascular Research 2010; 88(2): 34451. Epub 2010/06/15.

36. Gomes L, Fernando S, Fernando RH, Wickramasinghe N, Shyamali NL, Ogg GS, et al. Sphingosine 1-phosphate in acute dengue infection. PloS One 2014; 9(11): e113394. Epub 2014/11/20. 